\title{
TRAVELLING VIRTUAL BALISE FOR ETCS
}

\author{
A. FILIP \\ Faculty of Electrical Engineering and Informatics, University of Pardubice, Czech Republic.
}

\begin{abstract}
The railway industry has taken a great effort and is currently focused on exploitation of global navigation satellite system (GNSS) for the European train control system (ETCS). It has been assessed that replacement of track balises, used for safe train location determination, with virtual balises (VBs) detected by means of GNSS will significantly reduce the track-side infrastructure and operational costs. However, this innovated ETCS can be put into operations only in the case when detection of VBs by means of GNSS will achieve the same safety integrity level (SIL 4) and availability as it is required for physical balise groups (BGs).

This paper describes a novel travelling virtual balise (TVB) concept, which was proposed to meet the demanding ETCS safety requirements for GNSS using the existing European Geostationary Navigation Overlay Service (EGNOS) safety-of-life (SoL) service. The TVB concept profits from the basic feature of GNSS - i.e. the ability of abundant train position determination in GNSS service volume, which cannot be realized by current track balise groups (BGs) with a spacing of hundreds of metres or more. The frequent GNSS train positions are utilized for (1) fast diagnostics of on-board location determination system (LDS), (2) introduction of reactive fail-safety into LDS and (3) derivation and justification of the ETCS safety requirements for EGNOS.

The TVB concept brings one significant advantage to ETCS in contrast to the static VBs - i.e. the safety requirement for LDS doesn't depend longer on the distance between successive VBs. It means that the existing spacing between physical BGs (up to $2.5 \mathrm{~km}$ ) can be also preserved in case of TVBs. It can significantly improve the availability of LDS. Further it was found that a less demanding tolerable hazard rate (THR) requirement for GNSS of about $1 \mathrm{e}-7 / 1 \mathrm{~h}$ or more still enables to meet the ETCS THR requirement for VB determination, i.e. THR $\mathrm{VB}_{\mathrm{VB}}$ of $0.67 \mathrm{e}-9 / 1 \mathrm{~h}$. Thus the ETCS TVB concept opens the door for efficient use of the EGNOS SoL service, originally developed for aviation.
\end{abstract}

Keywords: EGNOS, ETCS, Galileo, GPS, high-safety integrity, LDS, railway signalling, reactive failsafety, safety-related systems, SIL 4.

\section{MOTIVATION}

Safe train position, velocity and time (PVT), among other basic quantities, are required for railway signalling. Nowadays all these quantities can be effectively provided by global navigation satellite system (GNSS). The very idea of the exploitation of global positioning system (GPS) satellites for signalling and train control is not new. The first experiments and field tests focused on train position determination using GPS and differential GPS (DGPS) were mainly performed in the United States and Europe more than 25 years ago. At that time the United States started work on the positive train control (PTC) system, which is based on GPS, and Europe chose the European train control system (ETCS), where track balises and on-board balise transmission module (BTM) were used for safe train position determination. Both PTC and ETCS have been successfully implemented on thousands of kilometres of tracks. 
Besides Europe, ETCS has also become successful in Asia - mainly in China, Turkey, Taiwan and South Korea. However, additional installations of ETCS with 'classical' track balises on heavy haul and other lines may not be in some countries efficient (Australia, Russia, China, etc.) - mainly due to high investment and maintenance cost of ETCS balises. The European Commission was aware of this potential ETCS limitation and therefore gradually initiated several R\&D projects oriented on the use of GNSS for signalling and ETCS in the 1990s.

There are two important dates regarding the use of GNSS for ETCS. Firstly, on 8 May 1998, a mixed train position determination solution by means of ETCS track balises and virtual GPS balises was described by Sterner [1]. Secondly, on 2 March 2011, the European Geostationary Navigation Overlay Service (EGNOS) based on GPS with its safetyof-life (SoL) service was officially declared available for safety operations in aviation. EGNOS belongs to the family of wide-area satellite-based augmentation systems (SBAS), which is similar to the following: US WAAS (RTCA DO-229 [2]), Japanese MSAT/QZSS and Russian SDCM. In spite of the fact that SBAS was originally developed and certified for safety operations in aviation, it also represents a strategic infrastructure for safetyrelated systems in land transport, including rail (Pullen et al. [3], Neri et al. [4]). It should also be mentioned that the European railway community also had a lot of expectations from nascent Galileo and its SoL service in the first decade of the new century. But finally the stand-alone Galileo SoL service was cancelled and it was decided to include Galileo in the multi-constellation and multi-frequency EGNOS V3, which should be operational around 2022.

Safe exploitation of aeronautical GNSS SoL service in railway signalling primarily means that the SoL service must be employed according to CENELEC standards (EN 50126 [5], EN 50128 [6], EN 50129 [7]). Excepting this, the GNSS SoL service must also be utilized effectively according to a realistic and generally acceptable strategy. The strategy says that GNSS should only replace the train location determination function, which is currently performed by means of track ETCS BGs and on-board BTM, a balise reader. Other ETCS subsystems, such as safe odometry (SIL 4), should remain untouched. And finally, there is a strong will in Europe to preferably employ the current well-working single-constellation EGNOS V2 (based on GPS) for ETCS and not to wait until 2022 for EGNOS V3. It is often said that 'what is sufficient for airplanes from viewpoint of safety, it must be also applicable for trains'. Later on Galileo or other constellations (GLONASS, BeiDou) could be used for availability of safety integrity improvement. In order to be able to think upon the EGNOS V2 exploitation for ETCS, it is necessary to make evidence first that EGNOS V2 is able to meet safety requirements for virtual balise (VB) detection. Due to this reason the travelling virtual balise (TVB) concept was proposed. It is demonstrated in this paper that the existing EGNOS SoL service, originally developed for safety operations in aviation, is using the TVB concept to meet the ETCS THR requirements for VB detection.

Differences in train position determination by means of physical BGs and VBs are outlined in Section 2. Section 3 briefly describes the allocation of ETCS tolerable hazard rate requirement to the VB and thus also for the entire LDS based on GNSS. Basic fail-safe principles applicable in railway safety-related systems compliant with SIL 3 and SIL 4 and their relation to the mono/multi-constellation LDS solutions are described in Section 4. Finally, the applicability of existing EGNOS V2 for the ETCS LDS with the reactive fail-safety architecture is justified by means of the so-called TVB and rapid LDS diagnosis in Section 5. The rapid and independent GNSS diagnosis undoubtedly represents the key to the LDS solution. 


\section{COMPRAIL}

\section{PHYSICAL AND VIRTUAL BALISES FOR TRAIN POSITION REPORTING}

In order to demonstrate the positive impact of the GNSS diagnosis on the ETCS safety integrity, a train position determination function within the ETCS using classical track BGs and VBs is outlined first.

The classical ETCS track BG, also called information point (IP), which shall be compliant with SIL 4 and $\lambda_{\text {IP }}$ of 1e-9/1 h (SUBSET-088 [8]), determines together with the on-board BTM the absolute position of a train. The ETCS odometry (SIL 4) provides the instant speed of a train and the relative distance from the last relevant balise group (LRBG) including its confidence interval $(\mathrm{CI})$. The train position, velocity and other data are reported via GSM-R to the track-side Radio Block Centre (RBC). One of the important odometry functions is called linking of balises via relative distance measurement. It is in fact an independent diagnosis of balises and on-board unit (ONB) because it enables detection of a deleted (missing) balise, incorrectly inserted balise or ONB fault.

In case of the VB concept the absolute position of a train is determined using the LDS based on GNSS. The instant position of the train is compared with the position of VBs whose coordinates are stored in the on-board European vital computer (EVC) and in RBC. If the actual GNSS train position together with the relevant CI match with a VB stored in the database, then the VB is considered as the last relevant virtual balise (LRVB) (see Fig. 1). The odometry together with the track database perform two following functions: (1) diagnosis of the consecutive VBs by linking with its direct positive impact on the desirable reduction of the safety integrity requirement for the GNSS LDS - i.e. GNSS THR increasing, and also 2) provision of the relative train position from the LRVB for ordinary train position reporting to the RBC. The relative train position is also provided when a VB is missed due to temporal GNSS signal-in-space (SIS) service outages or SIS shadowing in a harsh railway environment. The above described VB concept copies in fact the ETCS train position determination function using track BGs.

Now it should be said how ETCS can profit from GNSS. Let's compare for this purpose the diagnosis of train position determination in case of ETCS with track balises and diagnosis in the ETCS VB concept.

The ETCS ONB is able to perform fault diagnosis of physical BGs and also its own diagnosis only in locations of BGs. It is possible, thanks to BG linking, because position of next $\mathrm{BG}$ with respect to the $\mathrm{LRBG}$ position is known to the ONB and the correct $\mathrm{BG}$ detection can

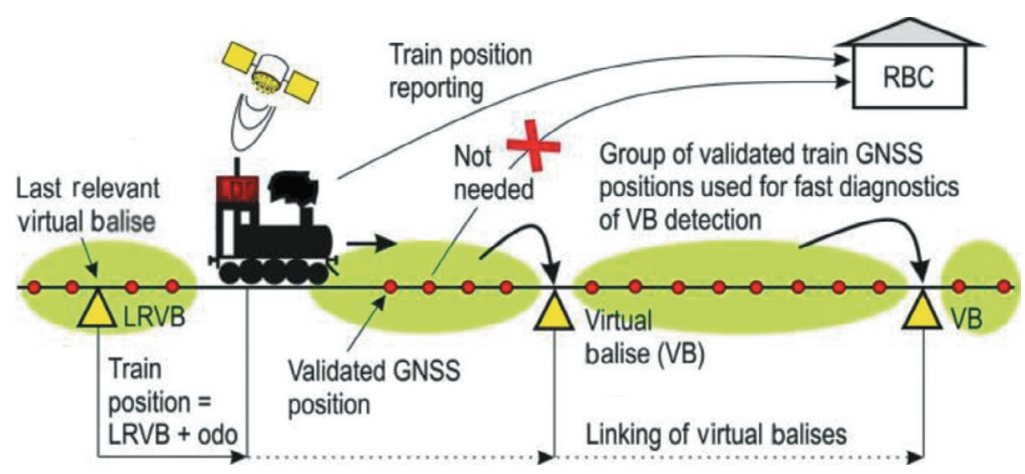

Figure 1: Exploitation of EGNOS SoL service within ETCS VB concept. 
be validated using the so-called expectation window (ExW). The ExW includes all potential uncertainties due to odometry and BG position errors. As it is depicted in Fig. 1, GNSS LDS is able to perform its fault diagnosis also in the vicinity of VBs or on the whole track section between VBs, depending on SIS visibility. It is important for LDS initialization (system start-up) in staff responsible (SR) mode or during entry into an ETCS area from an unfitted area. At this moment it is considered that the position of a train on parallel tracks can be determined using existing track elements such as track circuits, axle counters or balises. But in the future the safe parallel track discrimination function will also be performed by GNSS (Neri et al. [9]).

Abundant GNSS train positions outside the VB vicinity are not in fact needed under normal operation (after LDS initialization) for the train position reporting to RBC because it is provided by means of the relative distance measurement from the LRVB. However it is evident that these abundant GNSS positions together with the odometry data can be effectively used for the GNSS diagnosis and it can finally lead to reduction of safety requirements for the LDS based on GNSS.

\section{SAFETY REQUIREMENTS FOR VIRTUAL BALISE AND LDS}

The ETCS THR requirements for VB and GNSS LDS were derived by means of the ETCS core THR allocation in SUBSET-088 [8] and SUBSET-091 [10]. The target ETCS core THR of 2e-9/1 h/train is equally allocated to ETCS ONB (1e-9/1 h) and all ETCS track-side equipment. Then THR related to balise transmission hazard THR $_{\mathrm{BTX}}$ of $0.67 \mathrm{e}-9 / 1 \mathrm{~h}$ was determined. $\mathrm{THR}_{\mathrm{BTX}}$ was further sub-allocated to different track IP failure modes, such as balise deletion $\left(\right.$ THR $\left._{\text {BTX Deletion }}<3.3 \mathrm{e}-10 / 1 \mathrm{~h}\right)$, balise insertion $\left(\mathrm{THR}_{\text {BTX Insertion }}<3.3 \mathrm{e}-10 / 1 \mathrm{~h}\right)$ and balise corruption (THR BTX Corruption $_{1}<-11 / 1 \mathrm{~h}$ ). Since GNSS position is determined on board of a train, then only two following failure modes for VB were analysed: VB deletion and VB insertion (Filip and Rispoli [11], Filip [12]). These two VB failure modes can be described as follows:

- VB Deletion - means an event, when a VB (i.e. virtual IP) was not detected by means of on-board GNSS LDS. It can happen due to (1) excessive latent LDS error (wrong position) or (2) absence of train position in the GNSS LDS output. In both cases no VB is detected within the ExW provided by the odometry.

- VB Insertion - means an event when a wrong VB is determined due to wrong GNSS LDS position.

Since both VB failure modes are caused by a wrong GNSS LDS position (i.e. incorrect or no position), and diagnosis for both failure modes is provided by rapid and independent diagnosis in GNSS service volume, then the total THR $_{\mathrm{BTX}}$ of $0.67 \mathrm{e}-9 / 1 \mathrm{~h}$ was taken as THR for $\mathrm{VB}$, i.e. $\mathrm{THR}_{\mathrm{VB}}=0.67 \mathrm{e}-9 / 1 \mathrm{~h}$. $\mathrm{THR}_{\mathrm{VB}}$ determines in fact THR for the entire GNSS-based LDS, i.e. THR ${ }_{\text {GNSS LDS }}$, consisting of GNSS, odometry, etc. Tolerable hazard rate for single GNSS, i.e. THR $_{\text {GNSS }}\left(\mathrm{THR}_{\mathrm{SBAS}}\right)$, will be then derived for a given LDS architecture and for the most demanding LDS operational mode within ETCS. If we omit temporally speed restriction (TSR) mode, then the most demanding operational mode from viewpoint of safety for baseline ETCS is start of mission. It is because linking (diagnosis) of BGs cannot be used during this mode. Therefore start of mission will be used for derivation of $\mathrm{THR}_{\mathrm{GNSS}}$ - provided that safe parallel track discrimination can be solved using classical means, by two-tier GNSS augmentation (Neri et al. [9]), or by EGNOS V3 after 2022. 


\section{SINGLE- VERSUS MULTI-CONSTELLATION EGNOS FOR LDS}

Railway safety-related systems to be compliant with SIL 3 or SIL 4 must ensure that they will remain safe in the event of any kind of single random HW fault. This principle is known as fail-safety and can be achieved by means of the following techniques: inherent fail-safety, composite fail-safety or reactive fail-safety (EN 50129 [7] and Lovric and Gülker [13]). It is evident that implementation of these techniques determines not only the level of LDS safety achieved, but also how efficiently GNSS will be used within the LDS.

The inherent fail-safety technique allows a safety-related function to be performed by a single channel, provided that all the credible failure modes of the channel are not hazardous. It would be very difficult or impossible to make such evidence in case of complex SBAS and therefore inherent fail-safety is not further considered for the SBAS (EGNOS)-based LDS.

The composite fail-safety technique allows a safety-related function to be performed by at least two independent channels. Hazardous fault in one channel shall be detected and negated sufficiently quickly to meet the required THR. The fault is detected by the comparison of the output values of these two or more channels, or also by means of an additional independent diagnosis. This technique has been already employed in case of the dual-constellation EGNOS-R interface (see Fig. 2) (Filip [12], Filip and Rispoli [14]). The EGNOS-R was mainly proposed with the intention to meet THR of 1e-8/1 h (SIL 4) for SIS and simplify the required safety case and certification according to railway CENELEC safety standards. It was found that introduction of EGNOS-R also enables to reduce the EGNOS CI magnitude in the position domain.

Finally, the reactive fail-safety technique allows a safety-related function to be performed by a single channel, provided its safe operation is assured by fast detection and negation of any dangerous fault. The existing single-constellation EGNOS V2 itself can be considered as a system with reactive fail-safety, because the safety function is performed by the GPS, and its correctness is checked by the EGNOS infrastructure (see Fig. 3(a)). Nevertheless, the stand-alone EGNOS V2 is not yet able to meet the ETCS SIL 4 requirement for train position determination. It is because the position determination function must also meet the required

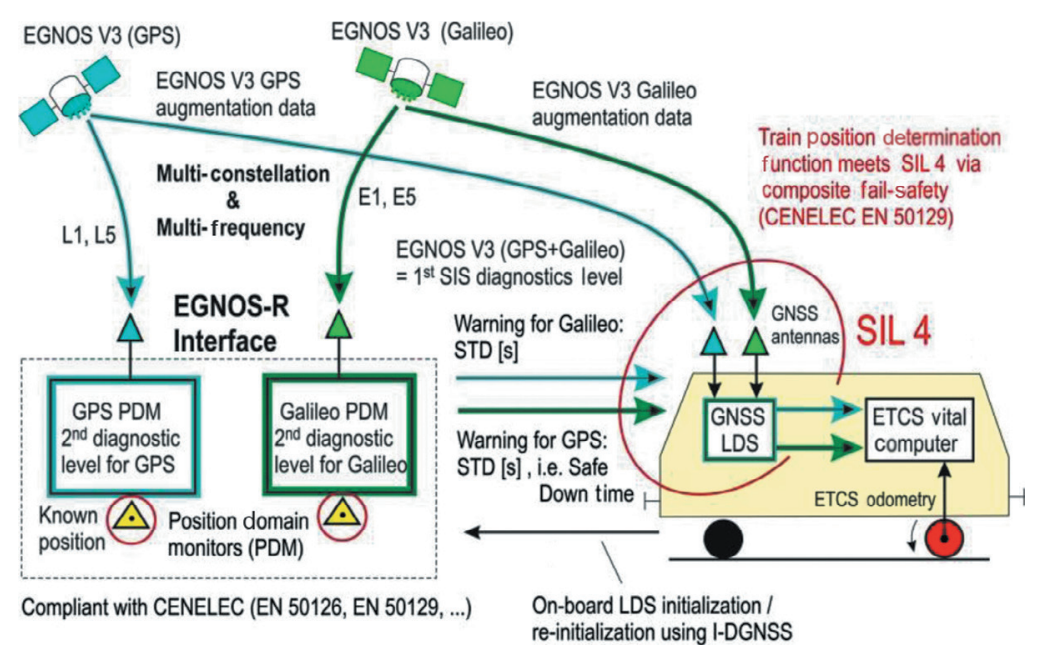

Figure 2: LDS based on dual-constellation EGNOS V3 and composite fail-safety. 

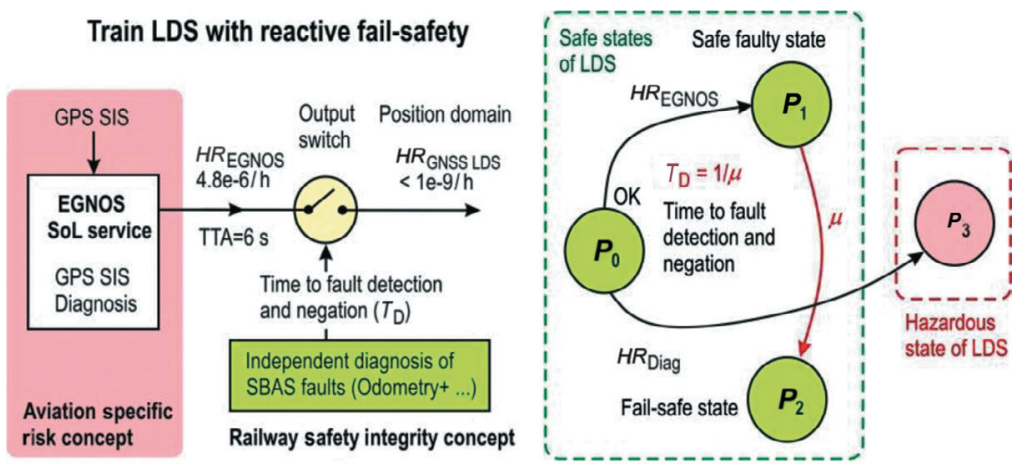

(a)

(b)

Figure 3: LDS based on single-constellation EGNOS V2 with reactive fail-safety: (a) Highlevel architecture. (b) Markov model.

integrity level in case of local effects, such as multipath, EMI, spoofing, etc., against which SBAS does not protect. That's why the SBAS fault diagnosis must be completed with an additional independent fault diagnosis realized, e.g. using the safe ETCS odometry (SIL 4), 3-dimensional track database (SIL 4) and other relevant techniques.

Markov model of the LDS based on single-constellation EGNOS and reactive fail-safety is depicted in Fig. 3(b). The following four system states are defined for the model:

- $P_{0}-$ Fully functional LDS state: both SBAS and independent SBAS diagnosis work well according to the specifications;

- $P_{1}$ - Safe faulty LDS state: SBAS is faulty and rapid diagnosis is functional;

- $P_{2}$ - Fail-safe state of the LDS: SBAS fault was detected and negated;

- $P_{3}$ - Hazardous LDS state: independent diagnosis of SBAS is faulty. Note: Although SBAS is functioning properly according to the specifications, the LDS is in a dangerous state.

The corresponding time-dependent LDS state probability $P_{1}(t)$ can be derived from the model as follows:

$$
P_{1}(t)=-\frac{H R_{\mathrm{SBAS}}\left[e^{-\left(H R_{\mathrm{SBAS}}+H R_{\text {Diag }}\right) \cdot t}-e^{-\mu \cdot t}\right]}{H R_{\mathrm{SBAS}}+\mathrm{HR}_{\text {Diag }}-\mu},
$$

where $H R_{\mathrm{SBAS}}$ - hazard rate of SBAS per $1 \mathrm{~h}, H R_{\text {Diag }}$ - hazard rate of SBAS independent diagnosis, $\mu$ - rate of diagnosis and fault negation. $P_{1}(t)$ is the safe faulty state probability in case of SBAS fault. Since $\left(H R_{\mathrm{SBAS}}+H R_{\text {Diag }}\right)$ is much smaller than $\mu$, eqn (1) can be simplified as follows:

$$
P_{1}(t) \approx-\frac{H R_{\mathrm{SBAS}} \cdot[1-0]}{-\mu}=H R_{\mathrm{SBAS}} \cdot T_{D}
$$

where $T_{D}$ is time to fault detection and negation, which is also sometimes called safe down time (SDT) (see (EN 50129 [7])). It is evident from eqn (2) that $P_{1}(t)$ depends on $T_{D}$ (i.e. on $1 / \mu$ ) and is no longer dependent on the time $t$. The corresponding LDS hazard rate during $1 \mathrm{~h}$ 
long mission can be expressed as $H R_{\mathrm{GNSS} L D S}=H R_{\mathrm{SBAS}} \times T_{D} \times 1 \mathrm{~h}^{-1}$. This relation will be further used for derivation of the ETCS THR requirement for SBAS/EGNOS in Section 5.

The conclusion: while composite fail-safety was mainly used for EGNOS safety integrity improvement to meet ETCS safety requirements for the LDS, reactive fail-safety implemented in the LDS is on the contrary indented for reduction of ETCS safety requirements for EGNOS. The reactive LDS solution exploiting the existing aviation EGNOS V2 SoL service is described below.

\section{FROM STATIC TO TRAVELLING VIRTUAL BALISE}

The baseline ETCS requires both track balises and ONB for safe train position determination. On the other hand GNSS estimates the position on board of a train. Let us assume that $\lambda_{\mathrm{ONB}}$ is the rate of occurrence of ONB being unable to detect a correctly working ETCS IP. If linking of IPs is active, then the duration of ONB failure corresponds to the time interval $T_{L}$ between two successive IPs marked as linked. Further if the average speed of a train is $v$ and the linking distance $D_{L}$, then the probability of ONB failure causing the IP deletion is

$$
P_{f, \mathrm{ONB}}=\lambda_{\mathrm{ONB}} \times T_{L}=\lambda_{\mathrm{ONB}} \times\left(D_{L} / v\right) .
$$

There is no safety requirement in respect of not being able to detect an IP when IP linking is active (see (SUBSET-088 [8])). As lately as two expected consecutive IPs announced by linking are not detected by ONB in the ExW, measured from the LRBG, the on-board vital computer shall consider the linking command of the second IP as a command to apply the service brake. Then the hazardous failure rate of ONB corresponding to the deletion of any IP met during one-hour-long mission is

$$
H R_{\mathrm{ONB}}=\lambda_{\mathrm{ONB}} \times\left(2 \times T_{L}\right) \times 1 \mathrm{~h}^{-1} .
$$

It should be mentioned here that balise insertion due to a wrong train position could have much more serious consequences; it could e.g. happen when system is initiated. In order to check the ONB functionality even before the detection of a regular and properly working BG by the ONB, an additional hypothetical 'testing' BG can be placed on the track ahead of the regular BG in the direction of movement from the LRBG (see Fig. 4). A much shorter ONB failure duration $T_{D}$ is achieved in this case. Equation (3) can be then modified as

$$
P_{f, \mathrm{ONB}}=\lambda_{\mathrm{ONB}} \times T_{D}
$$

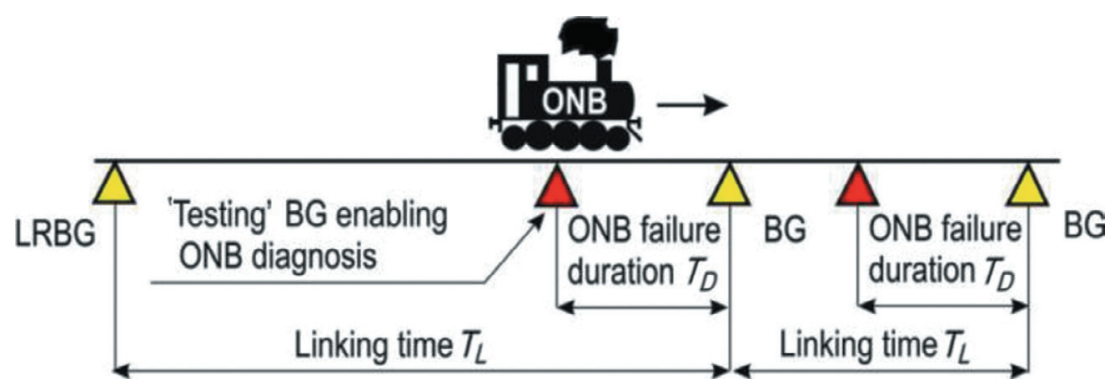

Figure 4: Diagnosis of ETCS on-board unit using 'testing' balise group. 


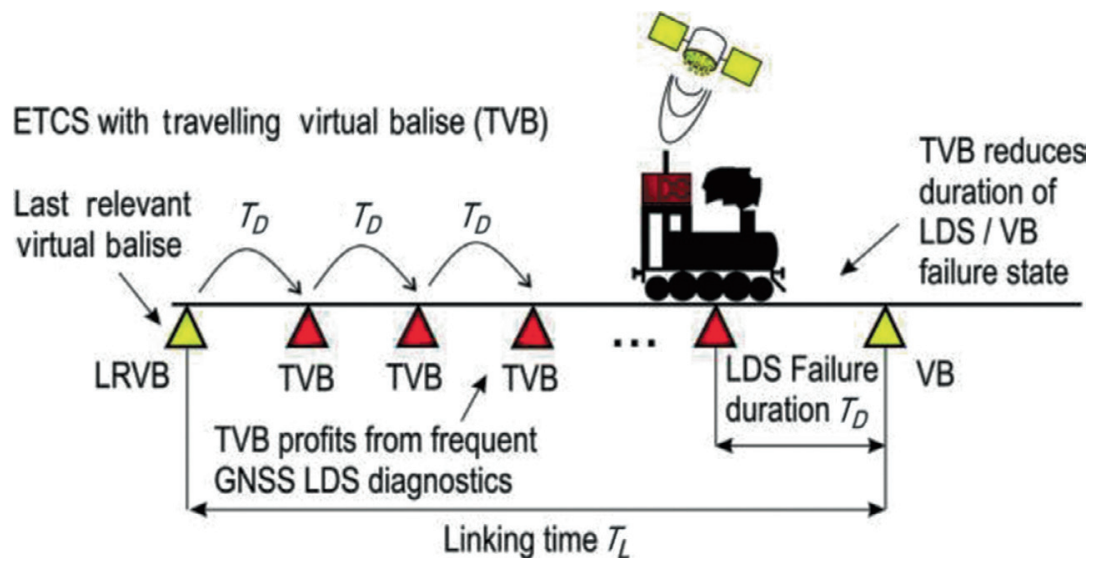

Figure 5: Travelling virtual balise introduced for justification of ETCS LDS architecture with reactive fail-safety.

and the corresponding ONB hazardous failure rate per mission $(1 \mathrm{~h})$ is

$$
H R_{\mathrm{ONB}}=\lambda_{\mathrm{ONB}} \times T_{D} \times 1 \mathrm{~h}^{-1} .
$$

Hazardous ONB failure rate related to IP deletion according to eqn (4) can be thus reduced with respect to HR in eqn (6) significantly. However it is evident that installation of the additional physical 'testing' BGs on a track would be very inefficient. Nevertheless, this reactive fail-safety principle can be easily implemented in case of the GNSS LDS. The 'testing' BG is simply replaced by the so-called TVB, as it is depicted in Fig. 5.

The TVB is equivalent to LRBG or LRVB from viewpoint of safety integrity because it is a validated GNSS train position using the independent diagnosis. The TVB arises from the LRVB as the validated subsequent GNSS train position generated just after the LRVB is detected and further travels to the next VB location in a given direction of movement. The TVB can also originate on a track section between two VBs during LDS initialization.

The detection function of the presence of an IP by ETCS on-board unit is a critical function and this function is the most critical when IPs are employed in such scenarios where linking is not used. It is e.g. during ONB initialization in SR mode or during entry into an ETCS area from an unfitted area when wrong IP can be inserted or IP can be deleted (missed). The ETCS THR requirement for GNSS must be derived using these scenarios considering that VB insertion could cause a more dangerous situation than VB deletion.

It is evident that the travelling VB can be utilized for the LDS diagnosis related to next VB deletion or insertion in the same manner as the hypothetical static 'testing' BG is used for the ONB diagnosis in Fig. 4. The ETCS THR requirement for GNSS, i.e. THR ${ }_{\text {GNSS }}$, can be determined for the LDS start-up from the THR requirement for VB deletion or insertion per mission, i.e. THR ${ }_{\text {GNSS vB }}$ of $0.67 \mathrm{e}-9 \mathrm{~h}^{-1}$ (see Sections 3 and 4), as follows:

$$
0.67 \mathrm{e}-9 \mathrm{~h}^{-1}=\mathrm{THR}_{\mathrm{GNSS}} * T_{D} * \mathrm{~h}^{-1},
$$

where $T_{D}$ is the duration of GNSS hazardous failure defined as the time interval between the two consecutive linked TVBs or linked TVB and next VB. Let's assume e.g. HR GNSs of $1 \mathrm{e}-7 / \mathrm{h}$ which corresponds to the SBAS integrity risk (IR) requirement for the aviation 
non-precision approach (NPA or en route). Then according to eqn (7) the acceptable hazard duration $T_{D}$ due to VB deletion/insertion is

$$
T_{D}=0.67 \mathrm{e}-9 / 1 \mathrm{e}-7 * 1 \mathrm{~h}=6.7 \mathrm{e}-3 \mathrm{~h}=24.12 \mathrm{~s} .
$$

It should be noted that the allowed horizontal alert limit (HAL) is quite large in this case, i.e. $0.3 \mathrm{nmi}(556 \mathrm{~m})$. HR of on-board GNSS receiver subsystem is neglected in this estimation.

The SIS IR of 2e-7/150 s for precision approach (PA) including LPV-200 operations is required in the vertical direction. Excepting this the SIS IR of 1e-9/150 s in the horizontal/ lateral (one-dimensional) direction shall also be met for the aviation PA operations. It seems that the integrity (i.e. guarantee) of accuracy in the horizontal plane or in the track direction would be sufficient for signalling in case of the reactive LDS architecture. Nevertheless, three-dimensional (3-D) track map appears as an effective means for the independent diagnosis of SBAS, and therefore the IR of 2e-7/150 was conservatively selected for signalling. The SBAS SIS hazard rate approximately corresponds to $4.8 \mathrm{e}-6 / 1 \mathrm{~h}$. Then the allowed duration of SBAS failure can be estimated as

$$
T_{\mathrm{D}}=0.67 \mathrm{e}-9 / 4.8 \mathrm{e}-6 * 1 \mathrm{~h}=1.36 \mathrm{e}-4 \mathrm{~h}=0.50 \mathrm{~s}
$$

The HAL of $40 \mathrm{~m}$ and VAL (vertical AL) of $35 \mathrm{~m}$ are required for LPV-200 operations, where the pilot's decision height is 200 feet $(60 \mathrm{~m})$ above the runway. The actual WAAS/ EGNOS accuracies $(95 \%)$ in horizontal/lateral and vertical directions are typically better than $1.1 \mathrm{~m}$ and $1.5 \mathrm{~m}$, respectively. The real SBAS performance in terms of SIS integrity is better than it is required by aviation. Let's consider the real EGNOS IR for of $6 \mathrm{e}-8 / 150 \mathrm{~s}$ for LPV I operations. Then the corresponding EGNOS SIS hazard rate is $1.44 \mathrm{e}-6 / 1 \mathrm{~h}$ and the acceptable duration of failure $T_{D}$ of $1.44 \mathrm{~s}$ can be estimated according to eqn (7). If SBAS receiver with an output rate of $10 \mathrm{~Hz}$ will be used, then all the above calculated values of $T_{D}$ are realistic - however under the condition that all SBAS failure modes will be properly detected and negated.

In spite of the fact that the TVB is not a priori defined by means of its geographic coordinates, it can efficiently substitute a number of static VBs with known positions and small spacing between them, which might be otherwise introduced for reduction safety requirements for GNSS, but might be also hardly detectable.

\section{CONCLUSION}

The travelling virtual balise was introduced into the ETCS VB concept with the intention to justify the exploitation of the existing aviation EGNOS V2 SoL service for the train LDS to be compliant with SIL 4 at a system level. The TVB concept fully supports the harmonization of the aviation and railway safety requirements for efficient use of the single EGNOS V2 SoL service for both aviation safety-critical systems and railway safety-related systems. Further the TVB ensures the continuity in the ETCS balise concept evolution oriented from the baseline ETCS platform with physical track balises to more efficient virtual ones stored in the ONB and track-side RBC.

The term 'TVB' has been proposed to reflect the analogy between the ETCS static 'testing' track BG and 'testing' VB intended for rapid fault diagnosis of the entire ETCS LDS. The adjective 'travelling' means that geographic coordinates of the TVB are not a priori assumption. The TVB propagates on the track section between two subsequent VBs, which can have the same spacing as the track BGs. The abundant GNSS train positions together with the 
odometry data and combined with other diagnostic methods, e.g. pseudo-range validation using a 3-D track database, are used for fast TVB validation for the required safety integrity.

The TVB is a validated train GNSS position that meets the THR requirement for VB deletion or insertion, i.e. THR ${ }_{\mathrm{VB}}$ of $0.67 \mathrm{e}-9 / 1 \mathrm{~h}$. The diagnosis of both LDS ONB and GNSS SIS mainly relies on TVB/VB linking. The TVB concept is fully consistent with reactive failsafety where the main channel itself, i.e. GNSS, may not meet the safety requirements, but the diagnostic channel must detect all possible dangerous failures so quickly that the overall safety target is met. The TVB brings the following additional benefits:

- TVB enables to preserve or even enlarge the VB spacing with respect to the maximum allowed ETCS BG spacing $(2,500 \mathrm{~m})$ without any impact on the entire LDS safety;

- Temporal TVB unavailability doesn't influence safety of train position determination because the safe ETCS odometry (SIL 4) is used for train position reporting in respect to the LRVB when it is required;

- Additional GNSS constellations such as Galileo, GLONASS or BeiDou can be used for LDS availability of integrity improvement;

- TVB efficiently substitutes a number of static VBs with small VB spacing;

- Introduction of the TVB doesn't influence the ETCS safety concept and the backward compatibility with the baseline ETCS is preserved.

\section{ACKNOWLEDGEMENT}

This work was supported from the European H2020 research and innovation programme within the RHINOS project (2016-2017).

\section{REFERENCES}

[1] Sterner, B.J., On the method of combining GPS and ETCS for localization purposes. The European Railway Research Institute (ERRI), 6pp., Draft of 8th May 1998.

[2] RTCA DO-229D - Minimum operational performance standards for GPS WAAS Airborne Equipment. RTCA Inc.: Washington DC, 2006.

[3] Pullen, S., Walter, T. \& Enge, P., Integrity for non-aviation users. GPS World, pp. 28-36, July 2011.

[4] Neri, A., Sabina, S., Rispoli, F. \& Mascia, U., GNSS and odometry fusion for high integrity and high availability train control systems. ION GNSS+ 2015, Tampa, 11 pp., 14-18 September 2015.

[5] CSN EN 50126 Railway Applications: The Specification and Demonstration of Dependability Reliability, Availability, Maintainability and Safety (RAMS). CENELEC European Standard, 2002.

[6] CSN EN 50128 Railway Applications: Communications, Signalling And Processing Systems - Software for Railway Control and Protection Systems. CENELEC European Standard, 2003.

[7] CSN EN 50129 Railway Applications: Safety related electronic systems for signalling. CENELEC European Standard, 2003.

[8] ETCS/ERTMS - Class 1, ETCS Application Levels 1 \& 2 - Safety Analysis, Part 3 THR Apportionment. SUBSET-088 Part 3, ISSUE: 2.3.0, 91pp., 2 April 2008.

[9] Neri, A., Capua, R., Salvatori, P. \& Palma, V., ERSAT EAV High Integrity Two-tiers Augmentation Systems, manuscript, 2016. 
[10] ETCS/ERTMS Safety Requirements for the Technical Interoperability of ETCS in Levels 1 \& 2. SUBSET-091, ISSUE: 3.3.0, 51pp., 8 May 2014.

[11] Filip, A. \& Rispoli, F., Safety concept of GNSS based train location determination system SIL 4 compliant for ERTMS/ETCS. Proceedings of ENC GNSS 2014, Rotterdam, 15pp., 15-17 April 2014.

[12] Filip, A., Multi-constellation railway SBAS interface: A common platform for advanced signalling compliant with SIL 4 world-wide. Proceedings of the International Heavy Haul Association 2015 Conference (IHHA), Perth, Australia, 10pp., 21-24 June 2015.

[13] Lovric, T. \& Gülker, J., Singe channel ATP architectures, a new trend in Europe? Proceedings of the World Congress on Railway Research (WCRR) 2001. Köln, 9pp., 25-29 November 2001. http://www.uic.org/cdrom/2001/wcrr2001/pdf/sessions/3_5/ 040.pdf

[14] Filip, A. \& Rispoli, F., SIL 4 Compliant train location determination system based on dual-constellation EGNOS-R for ERTMS/ETCS. Proceedings of the International Symposium on Certification of GNSS System (CERGAL 2014), Dresden, Germany, pp. 109-114, 8-9 July 2014. 